\title{
Study on Landslide Mechanism and Treatment Technology in Tunnel of Sandy Cobble Stratum
}

\author{
Li Borong ${ }^{1}$, , Yao Hongzhi ${ }^{1}$, Gong Juan ${ }^{2}$ \\ ${ }^{1}$ China Communications Construction Company Limited First Highway Consultants Co., Ltd, Xi'an, China \\ ${ }^{2}$ China Communications Construction Company Limited Third Highway Engineering Co., Ltd, Beijing, China
}

\section{Email address:}

1178981293@qq.com (Li Borong)

*Corresponding author

\section{To cite this article:}

Li Borong, Yao Hongzhi, Gong Juan. Study on Landslide Mechanism and Treatment Technology in Tunnel of Sandy Cobble Stratum. American Journal of Civil Engineering. Vol. 5, No. 5, 2017, pp. 307-314. doi: 10.11648/j.ajce.20170505.15

Received: September 1, 2017; Accepted: September 9, 2017; Published: September 22, 2017

\begin{abstract}
The steady ability of sandy pebble stratum is poor, during the tunnel construction, landslides occur frequently. In order to clarify the mechanism of landslide in sand gravel tunnel and put forward effective treatment technology, the paper takes the Gongboxia tunnel, which located in Qinghai Province, as the engineering background. In view of the landslide in tunnel, the influence factors and formation instability model are analyzed, also the comprehensive treatment technology of landslide is put forward. The results show that the geological factors are the main factors of tunnel collapse, design factors and construction factors contributed to the collapse of the tunnel collapse to a certain extent, the interaction of multiple factors; sand gravel stratum collapse mechanism belongs to loose medium caving, the instability mode is gravity collapse; based on the analysis of tunnel collapse mechanism. Put forward the "dense row of short pipe shed, three bench reservation core soil + locking anchor pipe, grouting backfill, the temporary support" comprehensive treatment technology, through on-site data reflect, have very strong practicability and pertinence. The research results can provide the material of engineering analogy for the design and construction of similar projects, and have important reference significance.
\end{abstract}

Keywords: The Tunnel, Sandy Pebble Formation, Landslides, Treatment Technology

\section{Introduction}

At present, the national highway network planning (20132030) has been introduced, road networks tend to be more perfect, the highway construction extending continuously in the west and mountains. At the same time, the special geological conditions also showed a trend of gradual increase in the tunnel construction. In east of QingHai area, the unfavorable geological conditions such as gravel and gravel are common in the tunnel.

The tunnel passes through the special stratum of sand and gravel. During the construction process, the collapse and difficult drilling will occur. At present, there are few researches on the use of mining method to pass through sandy cobble stratum. Lin J and Wang Y L took Ke Muqi tunnel as an example, the design and construction plan are optimized, and the construction technology and notice of tunnel excavation in sandy cobble stratum are expounded [1].
Ren $\mathrm{X} \mathrm{H}$ for the tunnel crossing the sloping sandy pebble excavation surrounding rock stability, the advance in the process of protecting measures, excavation method and tunnel through the pebble bed causes of landslides, using the finite element method (fem) was studied [2]. Leng $\mathrm{H}$ of pebble soil layer by using finite difference method in tunnel portal construction simulation, improved three step method is adopted to improve the construction is put forward, and to evaluate its usefulness [3].

To sum up, in the case of tunnel collapse in the construction of sand gravel stratum tunnel, the reason of tunnel collapse is mainly analyzed from the point of view of numerical simulation. However, the treatment measures of tunnel collapse are more dependent on construction experience, and few of them can be considered from the point of view of influencing factors of tunnel collapse and the mode of instability of surrounding rock. This paper takes the tunnel of sandy gravel stratum in QingHai 
Province as an example, starts with the mechanism of tunnel collapse, and then gives some engineering experience, and puts forward the targeted collapse treatment technology.

\section{Project Summary}

\subsection{Project Introduction}

Gongboxia tunnel is located in the Xunhua County of QingHai Province, along the ridge of the right bank of the Yellow River, import on the east side dam is about $1.5 \mathrm{~km}$ slope toe, exports in the dam area in about $1 \mathrm{~km}$ branch lander on the right bank slope bottom. The tunnel using line separate from left to right, the left line and pile ZK76 +912 ZK78 + 981, the right line all pile ZK76 + 930 ZK78 + 991 total length of $2061 \mathrm{~m}$, all belong to long tunnel, tunnel maximum depth of about $221 \mathrm{~m}$ (left), $218 \mathrm{~m}$ (right). Among them, the tunnel to import $584 \mathrm{~m}$ across blunt pluvial sandy pebble stratum, maximum depth of $75 \mathrm{~m}$ sand pebble layer location.

\subsection{Engineering Geology}

Gongboxia tunnel is located in the XunHua basin, no fault structure is adopted, and strata are monoclinic. strata occurrence in between $150-165^{\circ} \angle 5-10^{\circ}$. The import section belongs to the river erosion accumulation landform, micro landform belongs to the the Yellow River River impact accumulation terrace, terrain is relatively slow and round. In addition, the bedrock rock in silty mudstone with sandstone and conglomerate, silty mudstone, strong weathering rock, soft rock is very thin, $\sim$ thick layered structure, fracture, interlayer cataclastic structure rock was poor, poor integrity. The formation water is almost zero pebble gravel as main component of gneiss and granite gneiss, sandstone, the egg stone hard, not weathering, and the presence of large boulders, the silt and sand filling, filling the local enrichment.

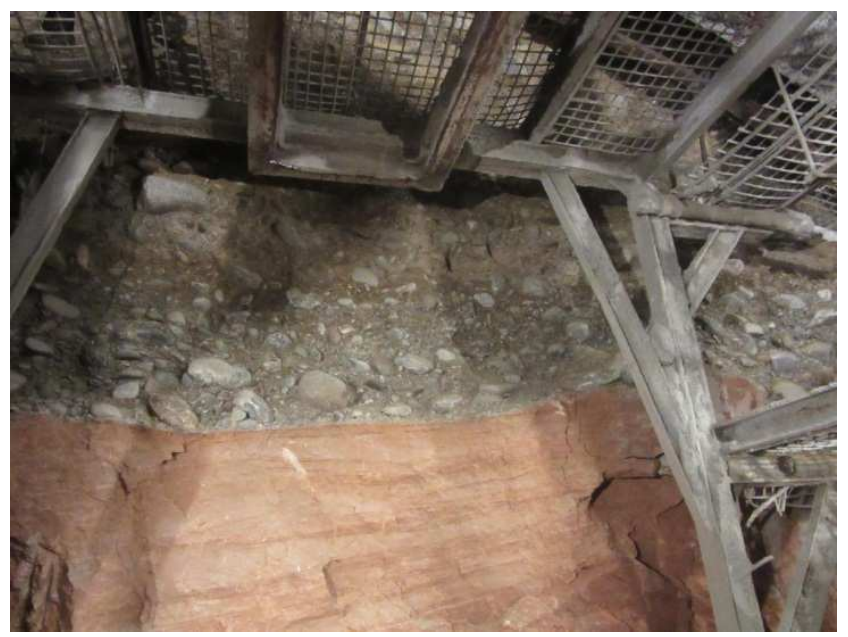

(a). Hydraulic jump in longitudinal section.

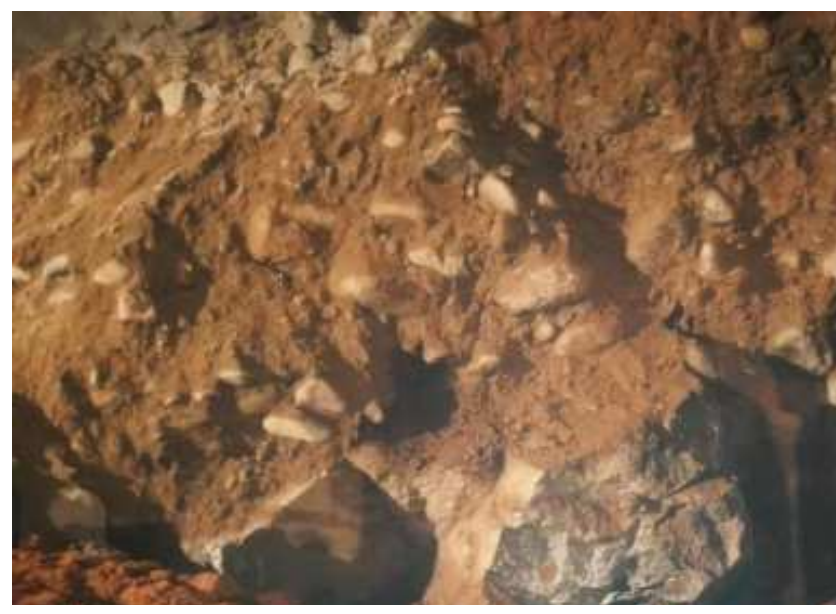

(b). Pressure forces acting on the surface of the control volume

Figure 1. Tunnel sandy pebble distributions.

\section{Tunnel Collapse}

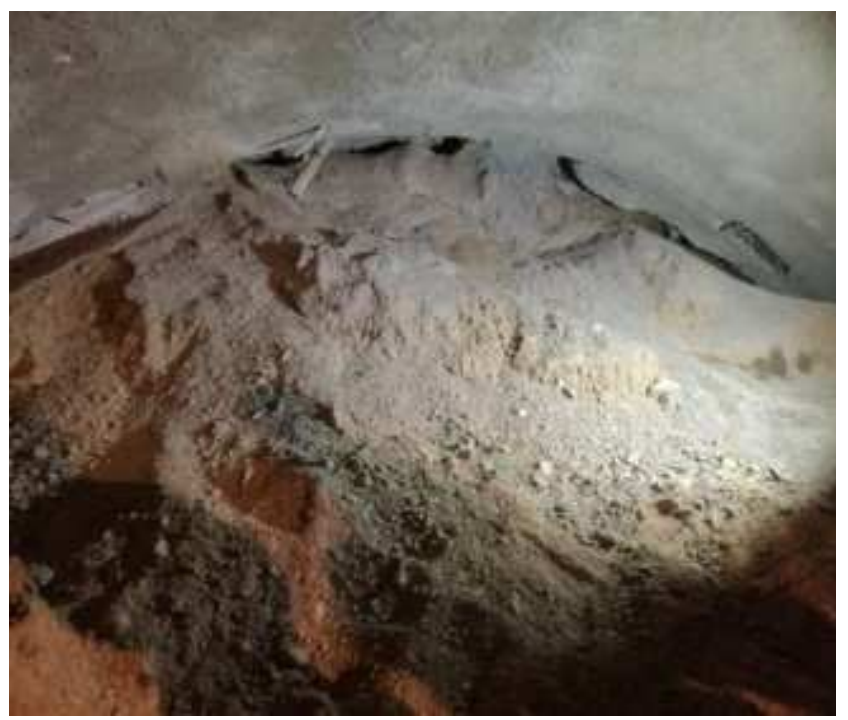

(a). Arch collapsed.

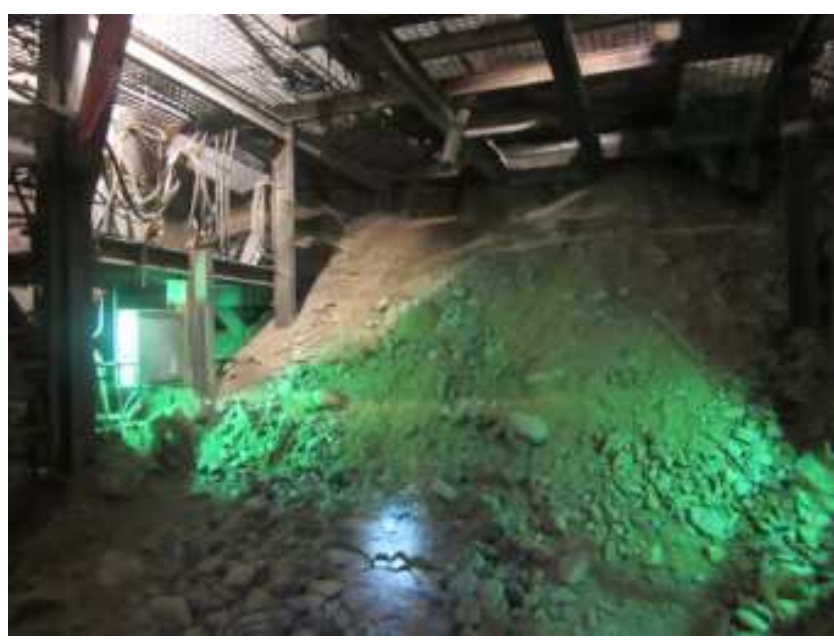

(b). Constraints collapsed 


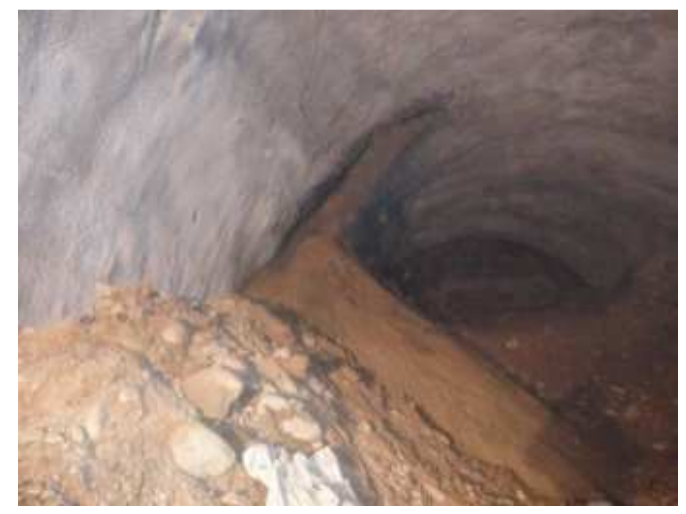

(c). Hance collapsed.

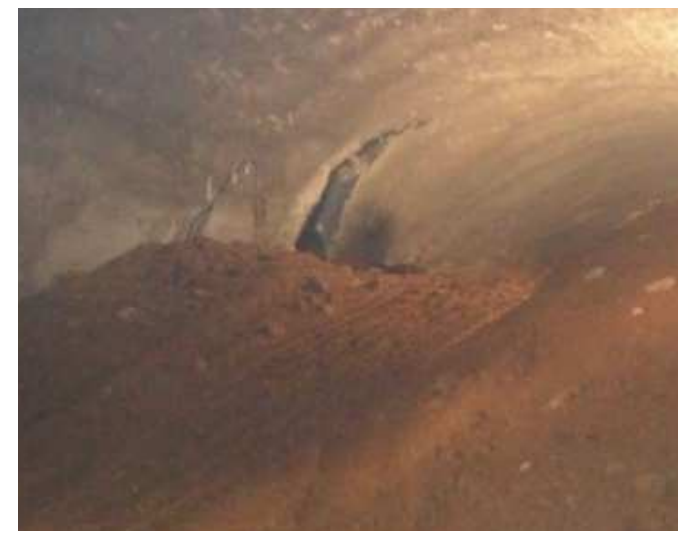

(d). The initial supporting cracking.

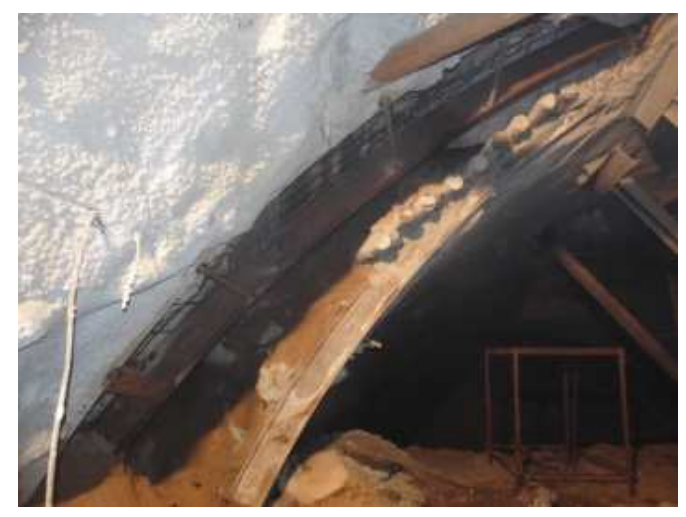

(e). Primary support invasion

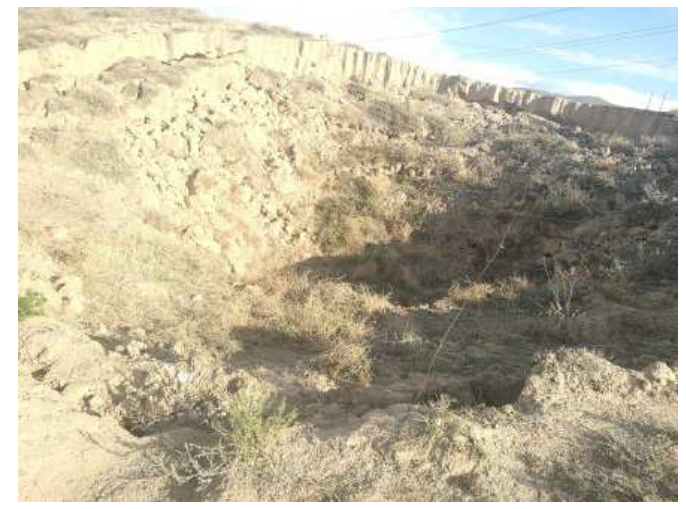

(f). The surface of the roof.

Figure 2. Landslides during tunnel construction
Because of the unique engineering characteristics, the landslides occur frequently in tunnel during construction. Through the statistics of the Gongboxia tunnel during the construction of many landslides accident analysis, landslides occurred arch and side wall in the tunnel roof, the displacement of large sometimes wear to the surface collapse. Landslides can be divided into following categories: vault collapse, collapse, collapse, side wall cracking, the initial support of initial support and serious invasion of the ground collapse.

Landslide case description: tunneling to ZK76+965, in the original advance ductule, under the support of excavation section, vault above has a small amount of residue in drop, backward evacuated, construction personnel found the situation changes, falling after half an hour and then dome. Like sand flow landslides happened, 2 minutes duration. Landslide section height to the surface, about $30 \mathrm{~m}$, the collapse of the long time, directly affected to the surface, causing the three constraints in front surface roof caving, the biggest one is to form a diameter of $4.5 \mathrm{~m}, 1.5 \mathrm{~m}$ deep funnel-shaped cavity collapse.

With the development of tunnel tunneling, arch excavation, due to mechanical disturbance, face slip, core soil sudden instability, outward extrusion, lead to the collapse of the tunnel face soil inside tunnel face was completely collapsed covered collapse with gravel loose fine sand, amount of landslide is about $45 \mathrm{~m}^{3}$. In addition, the tunnel will occur landslides on both sides, serious a landslide occurred in the tunnel on the left side of the wall, on the left side of the wall location steel arch bending deformation, steel mesh, spray at the beginning of $\mathrm{C} 25$ concrete floor crack, crack width is 5 $35 \mathrm{~cm}$, toroidal length is $1.2 \sim 1.5 \mathrm{~m}$, collapse of the tunnel section with a third space. As a result, the initial support showed a sharp deformation and penetrated the clearance of the secondary lining.

\section{Mechanism Analysis of Tunnel Collapse}

Landslide is a typical instability phenomenon, which is caused by the combination of geological force, engineering force or two kinds of forces, resulting in displacement, deformation and even destruction of some or all of the surrounding rock. A large number of engineering practice shows that the stability of tunnel surrounding rock not only with the original stress state of surrounding rock, the broken degree, structure characteristics and the effects of groundwater conditions, but also with the tunnel construction method and supporting time and related forms [4].

Song B Through the study found that there are many influence factors in tunnel collapse, generally speaking, there are factors such as geological factors, design factors, construction factors [5]. Combined with the field construction of Gongboxia tunnel, found that the formation is loose, the cohesive force between the particles is low, and it is easy to be disturbed. 


\subsection{Influencing Factors of Tunnel Collapse}

\subsubsection{Geological Factors}

Sandy gravel formation is a typical mechanical unstable stratum, which is loose structure, poor arching, high permeability and low self stabilization. There is no cohesion between the particles in the formation. In the anhydrous state, the gravel particles pass through the point to point force, and the formation reaction is sensitive and easy to be disturbed.

During tunnel excavation, the surrounding rock mass is disturbed and the stress is released. As the free face of the plane has no support, there is a trend of deformation to the tunnel, so that the excavation of the tunnel has a tensile stress. Coupled with the loose stratum, cohesion is low, can not afford or can only bear the tensile stress is small, the stress concentration generated at the fracture surface, followed by stress redistribution, it will produce new fracture surface, finally under the action of self weight, and new surface rupture along the primary slip, the wrong move, until the collapse.

The distribution of pebble particles in the formation is uneven, and the specific gravity of each area varies. In the construction of roof and tunnel face surrounding rock loose, poor stability, low shear strength if break the stability of surrounding rock will collapse. On both sides of the tunnel excavation because before and after different degree disturbance, can also cause the arch waist or side wall collapse, collapse in the form of direct action of gravity of primary support structure, the role of the primary support deformation of surrounding rock pressure and loose rock pressure increases, causing the initial support of cracking and invasion.

\subsubsection{Design Factors}

Because the geological exploration work of the earlier stage is not in place, the geological condition of the tunnel site area is not clear enough, and the supporting measures adopted by the design are not effective. Very clear: the original design of the forepole $\varnothing 42 \times 4 \mathrm{~mm}$, length is $4 \mathrm{~m}$, extrapolation Angle for $10 \sim 15^{\circ}$ small duct grouting in advance. In site construction, found that the scope of reinforcement is limited, the supporting effect is bad, can't contain vault collapse of the basic problem, also a big roof collapsed.

In addition, the understanding of the engineering characteristics of sandy gravel formation is not enough, and the construction method is often based on engineering analogy and engineering experience. The construction method of the original design for the single side drift method, in the process of implementation, the excavation of the left side heading, not in the next construction of temporary support, found on the right side of sand and gravel to the left until the collapse slide. Finally, it is proved that the method is not feasible.

\subsubsection{Construction Factors}

During construction, the construction speed and construction quality will cause tunnel collapse. The stability of formation surrounding rock is poor and can not be stable for a long time. If the excavation is fast and the support is relatively slow, sometimes the best supporting time is missed due to the neglect of consciousness, resulting in excessive deformation of the tunnel, and then collapse. Too rush deadlines, resulting in poor quality of construction, behind the support structure not dense or supporting hole, can not make supporting structure and surrounding rock closely, can not play the bearing capacity of the two, resulting in the plastic zone of the surrounding rock to expand, and the possibility of instability collapse.

Moreover, the impact force and vibration intensity of excavation reflect the disturbance degree of surrounding rock, and the greater disturbance, the more landslide likely cause. At the same time, the construction staff awareness of security is not strong, as well as poor quality awareness, but also cause landslides.

In conclusion, the geological factors are the main factors of tunnel collapse.

\subsection{Sandy Pebble Stratum Instability Mode Analysis}

Guan B S studies the mechanism of landslides, the tunnel landslide is divided into sliding type dangerous rock, loose medium caving variant and hard rock, soft rock creep rock burst type [6]. Loose sandy pebble stratum and weak no bond or bond, belong to a loose medium type caving.

Sandy pebble stratum deformation and failure is mainly affected by cementation between particles and the formation of loose degree is decided by such factors as the main damage form is given priority to with gravitational collapse. Deformation mechanism is due to tunnel excavation disturbance, surrounding rock stress release. At this time, when the tensile stress of the tunnel surface is greater than the cohesive force between the particles, the fracture will occur and extend to the interior of the surrounding rock to form a fracture surface. Under the continuous disturbance, surrounding rock stress redistribution and continuously produces new fracture surface, under the action of gravity stress and tectonic stress, the native and fracture surface sliding, dislocation, until the collapse, a cave arch is formed within the surrounding rock, to a temporary state of balance. Chen S M et al think: excavation tunnel in loose medium, if not timely support, the cavity at the top of the rock and soil body will collapse, until form a cave of parabolic arch, the upper cavity geotechnical weight borne by collapsing arch [7-8].

Gongboxia tunnel, the surrounding rock is loose and cementation is poor. The excavation, without timely support, the vault top soil along the stress due to rupture of unbalance, collapsing into a dome shaped arch, into a state of equilibrium. If this stratum is disturbed, the arch will continue upward collapse, serious condition, soil will collapse, rupture will extend to the surface, until the roof. Similarly, if the collapse occurred in the side wall, the instability mode is also so, according to the different degree of disturbance, the formation of the corresponding arch collapse. 


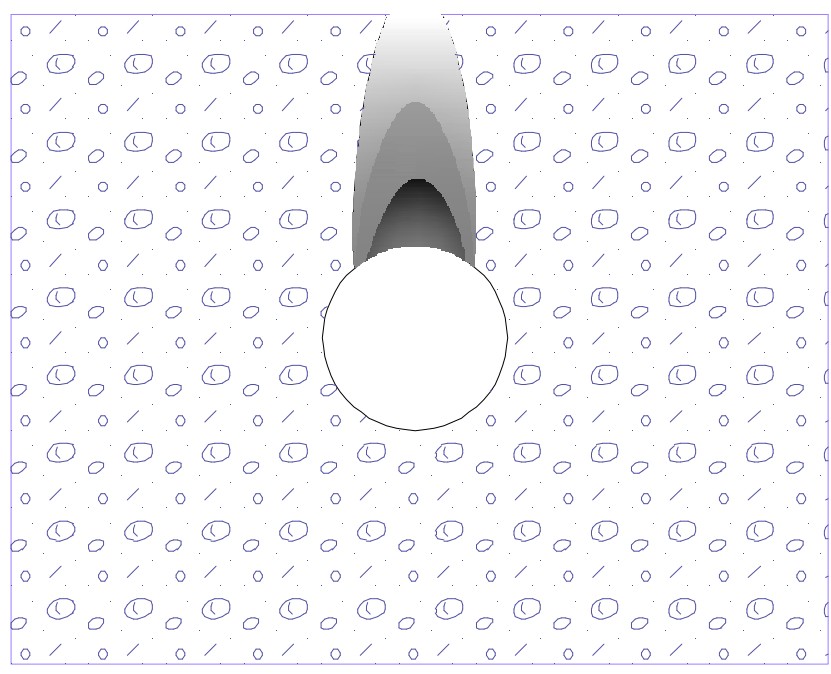

Figure 3. Sand gravel strata excavation instability.

\section{Sandy Pebble Formation Technology to the Treatment}

In the tunnel collapse, landslide location mainly concentrated in the vault, constraints, wall collapse. Some complicated landslides and relatively more difficult. However, the collapse mode of sandy gravel stratum is mainly gravity collapse, the surrounding rock is relatively loose, the cementation is poor, the self stability is weak, and without support, it will collapse soon. Therefore, if we want to successfully cross the sand and egg layer, we should be aware of the mechanism of the collapse of the premise, the anti collapse attention should be placed on supporting measures to prevent landslides.

\subsection{Dense Exhaust Pipe Roof}

The original design of the advanced small pipe support basically can't solve the problem of arch collapse. Then, turning to pipe roof support. Due to large proportion of sand pebbles, loose and good grading, prone to collapse when drilling holes, drilling difficulties and other issues. Conventional design parameters of pipe roof cannot apply to sandy pebble stratum, will be the end of pipe roof problem such as the offset is too large. Through field test, finally decided to adopt close short pipe roof support. "Close" refers to the ring between the pipe roof to the relatively small spacing, arrangement more closely. "Short" refers to a short length of pipe roof. The supporting parameters are shown in table 1 .

Table 1. Exhaust pipe roof design parameters.

\begin{tabular}{lllll}
\hline Pipe roof & Length & the ring to the distance & Angle of extrapolation & The lap length \\
\hline $\begin{array}{l}\text { Hot rolled seamless steel tube } \\
\text { Hot }\end{array}$ & $6 \mathrm{~m}$ & $15 \mathrm{~cm}$ & $15^{\circ}$ & $1.5 \mathrm{~m}$ \\
\hline
\end{tabular}

Close short pipe roof at a certain distance, into a fanshaped arrangement in the tunnel arch department, not only beam arch effect, and improves the cohesive force, improve the surrounding strata. Close short pipe roof formed toroidal reinforcement ring radial well constraints surrounding strata and prevent its pressure relief and relaxation, ensure the safety of the excavation process. Basically contain arch above the tunnel flow sand and collapse phenomenon, the supporting effect is shown in figure 4.

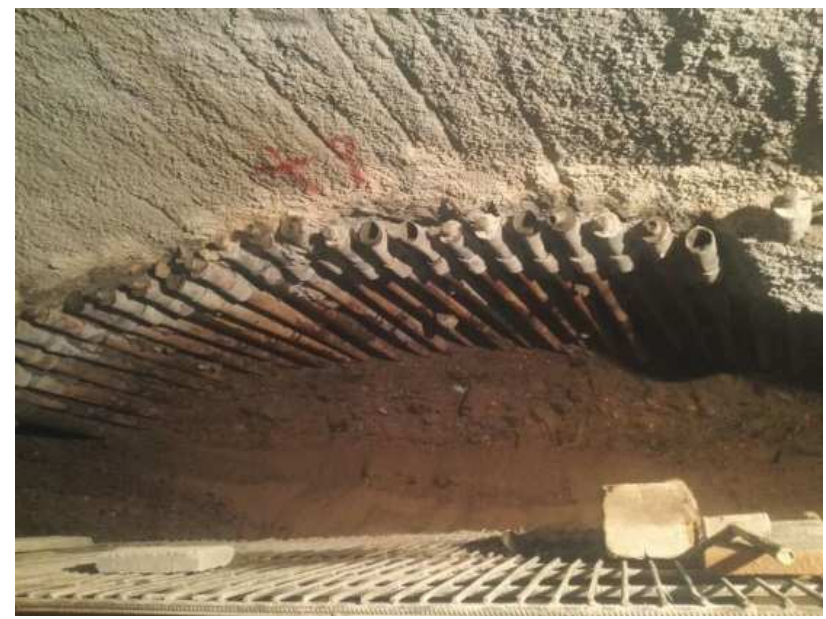

Figure 4. Close the short pipe roof supporting effect on site.

\subsection{The Reserved Core Making Three Steps + Lock Foot Anchor Pipe}

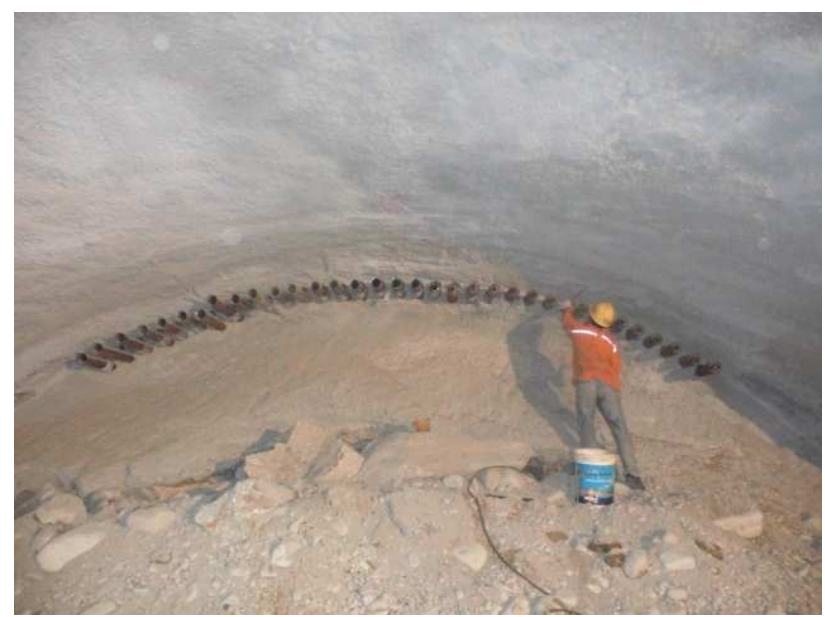

Figure 5. Core soil constraints set aside.

The original design of the construction method for single side heading method, but found in site construction, the feasibility of this method to implement more bad, the landslide problem is serious. Finally, changed to be three steps aside core construction method.

In close short tube shed advance support under the premise of first to advance guard and vault to pre-reinforcement of 
surrounding soil, circular excavation steps again, and obligate core soil, timely support after excavation, and then, steps and down the steps in the excavation, excavation and supporting, form a line production. Step method is proposed, which can conduct multiple working face, rapid construction, timely closed loop, play together the bearing capacity of the surrounding rock and lining. Among them, the reserved core soil (figure 5) can enhance the stability of the constraints, the constraints to prevent collapse of instability.

Because there is no middle-board in the three step excavation method, the vertical displacement is larger, and the vertical deformation control mainly depends on both sides of the arch foot. This shows that the stability of the arch foot is critical. During the construction of the anchor pipe inserted by two feet (material specifications and close short pipe roof), close to the level into the surrounding rock, in 4 $6 \mathrm{~m}$ in length, average arrangement in two common steel arch frame $(50 \mathrm{~cm})$, connected by transverse reinforcing steel bar and steel arch in the middle. Thus, to improve the bearing capacity of the arch foot. Moreover, through the lock foot anchor pipe grouting (figure 6), also can have improve on both sides of the cohesive force and the stability of surrounding rock.

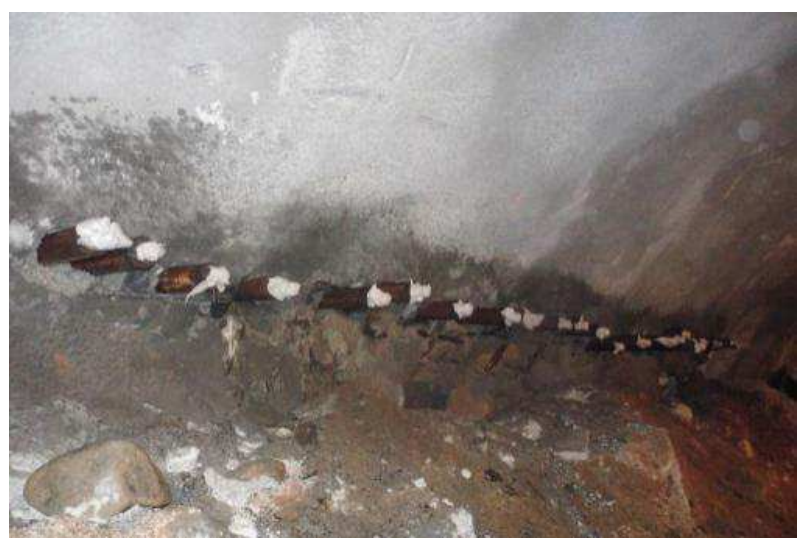

Figure 6. Lock foot anchor pipe.

\subsection{Grouting Backfill}

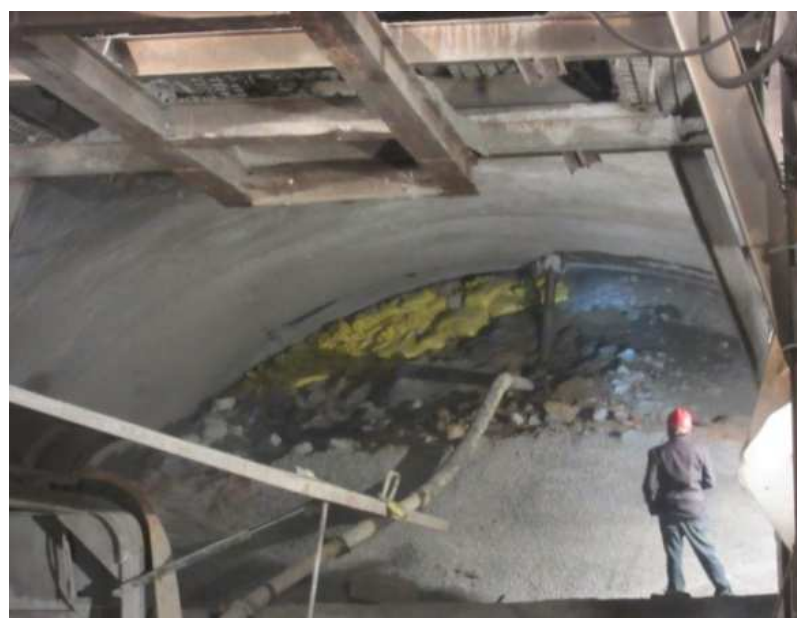

Figure 7. Sandbag plugging and backfill of the grouting.
Beginning in the vault for pipe shed advance support, drilling process, can cause disturbance to the surrounding rock is too large, broke the formation of equilibrium state, part of the surrounding rock instability, the phenomenon of sand flow and landslides. In order to prevent further landslides, the scene processing measures are generally use sandbag first block, then turn landslides in pumping cement mortar, backfill (figure 7). In addition, if the wall of rock mass is poorer, more loose. Sometimes occur landslides, processing measures are to walk the perimeter of the cavity collapse residue, to put 2-3 m of cement mortar, backfill, fill the cavity collapse in time ( figure 8 ).

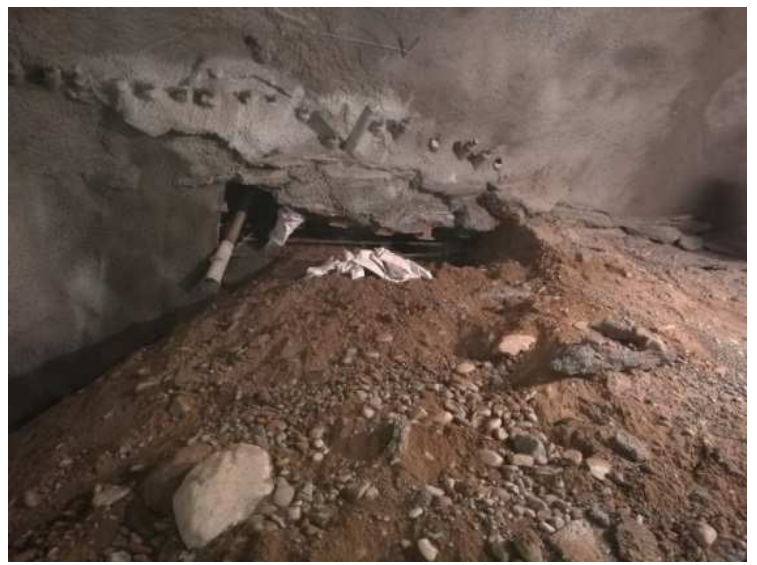

Figure 8. Cavity backfill grouting.

\subsection{Temporary Support in the Hole}

Through sand gravel stratum, the stratum is loose, the arching sex is bad. Jiang Y C et al think that loose surrounding rock pressure in surrounding rock pressure is given priority to [9-10]. in the surrounding rock loose collapsing the gravity or the weight of direct effects on the tunnel lining, can lead to deformation of primary support, serious when primary support cause craze. Primary support to prevent further deformation, deformation of serious parts inside the hole, using I20a type ibeam for temporary support, in order to change the stress of surrounding rock and initial support, improve the stability of surrounding rock, prevent collapse of surrounding rock and secondary collapse (figure 9).

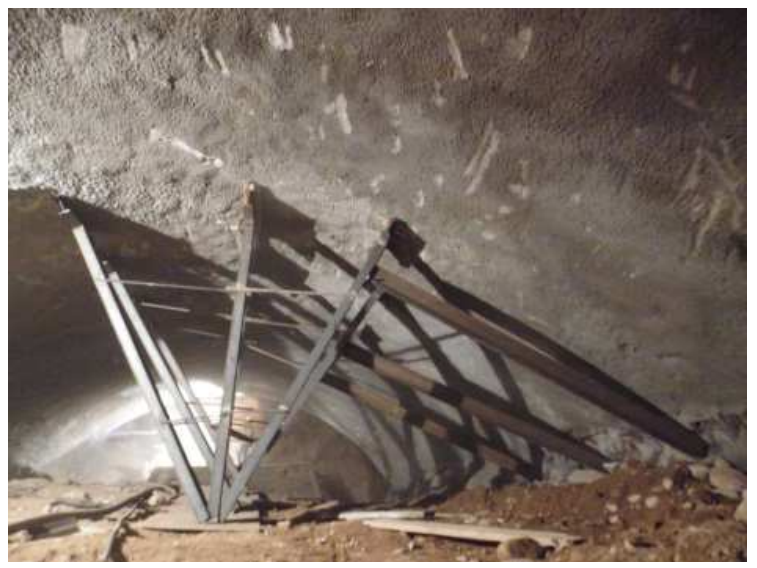

Figure 9. Temporary support in the hole. 


\section{Treatment Technology Evaluation}

In order to accurately and truly understand the effect of tunnel collapse treatment, The monitoring data of representative section $\mathrm{ZK} 77+220$ in sand gravel area is selected and analyzed. Deformation and the change of time curve is shown in figure 10.

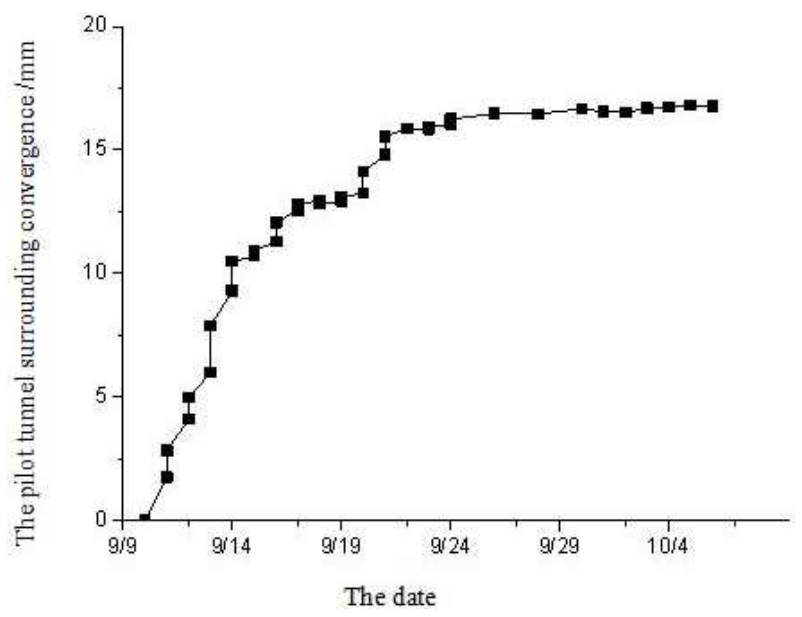

(a). ZK77 +220 pilot tunnel on peripheral convergence curve over time

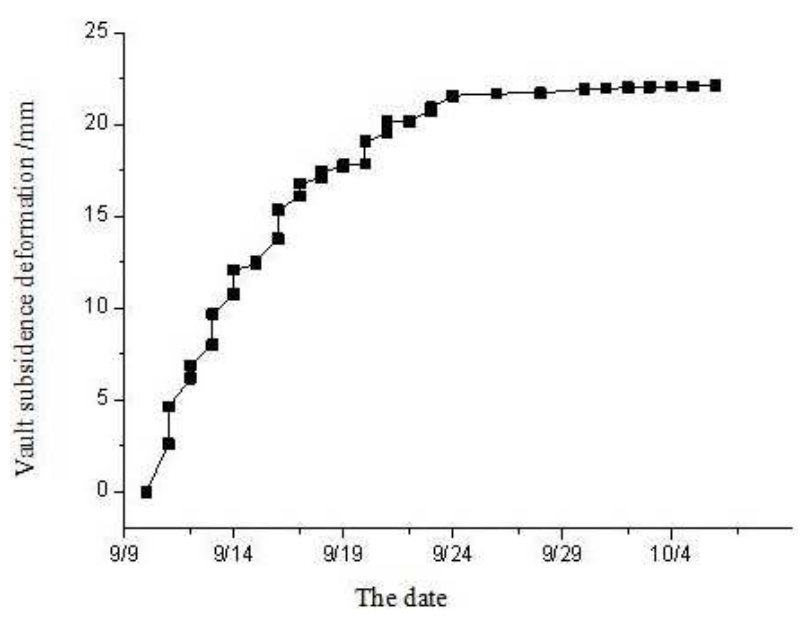

(b). ZK77 +220 vault subsidence value changing with time curve

Figure 10. Relation curve of deformation - time.

Can be seen from the figure 10 , the section ZK77 +220 peripheral convergence value and vault subsidence probably in two weeks without rising trend, began to convergence, basically stable. And peripheral convergence value below 20 $\mathrm{mm}$, vault sink the accumulative deformation under $25 \mathrm{~mm}$, within the prescribed limits. This shows that the tunnel collapse treatment technology used in Gongboxia tunnel (dense short pipe shed, three steps reserved core soil + anchor bolt pipe, grouting backfill, temporary support in the cave) is effective and practical.

\section{Conclusion}

One of the most serious problems encountered when tunneling through sandy cobble formations is tunnel collapse.
Taking the Gongboxia tunnel as an example, this paper summarizes the tunnel collapse, and analyzes the mechanism of the tunnel collapse. On this basis, a comprehensive treatment technique for collapse of tunnel through sandy gravel area is put forward, and the technical evaluation of landslide treatment is carried out in combination with monitoring data of monitoring in tunnel. The effect is obvious, and the following conclusions are obtained:

1. In the process of construction, sand and gravel tunnel collapse situation encountered: vault collapse, collapse, wall collapse. In addition to the direct collapse, the collapse of the tunnel will also cause initial support cracks, initial support limits and roof fall in severe cases. Through the sand gravel stratum tunnel collapse problem is the result of many factors: the geological factors are the main factors of tunnel collapse problem, design factors and construction factors exacerbated the problem of tunnel collapse in different extent. Design factors and construction factors belong to human cognition, and they are inevitable human factors.

2. Sand and gravel strata landslide belongs to loose medium caving, the instability mode is gravity collapse, destruction form of arch collapse form, and this is only temporary equilibrium arch collapse, once the disturbance occurred in two landslides, to reach a new equilibrium state.

3. Based on the analysis of influencing factors of tunnel collapse and strata instability mode, put forward the "comprehensive densely short shed, three bench reservation core soil + locking anchor pipe grouting and backfill, the temporary support of the collapse treatment measures. From the data of field convergence and vault settlement, the comprehensive treatment technique is very practical and can provide reference for the design and construction of the tunnel engineering.

\section{References}

[1] Lin jiang, Wang Yong-liang. Introduction to branch wood its tunnel sandy pebble stratum construction treatment [J]. Journal of highway tunnel, 2016 (1): 57-59.

[2] Ren Xiao-hui. Construction process of surrounding rock of tunnel crossing the sloping sandy pebble numerical analysis [D]. Dalian jiaotong university, 2013.

[3] Leng Hao. Sandy pebble soil layer of mountain tunnel portal construction mechanics research [D]. Southwest jiaotong university, 2016.

[4] Liu Biao. The study of stability of rock tunnel surrounding rock [D]. Southwest jiaotong university, 2014.

[5] Song Bo. Da shan highway tunnel collapse mechanism and treatment measures of research [D]. Chengdu university of technology, 2015.

[6] Guan Bao-shu. Tunnel project construction points set [M]. People's traffic press, 2011.

[7] Chen Shui-mei. The roof of the underground engineering excavation adit to collapse arch shape and surface subsidence rule analysis [D]. Hefei university of technology, 2015. 
[8] Wang Yi-cai. Tunnel project - second edition [M]. People's traffic press, 2015.

[9] The ministry of communications of the People's Republic of China. Highway tunnel design specification [S]. Beijing: people's traffic press, 2016.
[10] Jiang Ying-chao. The disturbance mechanism of shield tunneling in sandy pebble formation research [D]. Southwest jiaotong university, 2014. 